\title{
Gut throughput dynamics in the appendicularian Oikopleura dioica
}

\author{
Ángel López-Urrutia ${ }^{1, *}$, José Luis Acuña ${ }^{1}$ \\ ${ }^{1}$ Area de Ecologia, Departamento de Biología de Organismos y Sistemas, Universidad de Oviedo, C/Catedrático Rodrigo \\ Uria (s/n), Oviedo 33071 , Spain
}

\begin{abstract}
Oikopleura dioica is an excellent model for studying food flow through the digestive system because of its transparency, non-motility and because fecal pellets move along the digestive system in an orderly sequence which can be easily timed. By observing fecal pellet circulation within the gut of healthy individuals, we have concluded that the average number of fecal pellets inside the gut of O. dioica is $2.878 \pm 0.015$ (mean $\pm \mathrm{SE}, \mathrm{n}=43$ ). Thus, gut passage time (GPT, min) can be estimated from the time interval between successive fecal pellets (DI, min fecal pellet ${ }^{-1}$ ) as GPT $=2.878$ DI. This establishes the basis for estimating GPT from simple fecal pellet production rate incubations, and is one way of determinating GPT without manipulating food concentration or quality, a major shortcoming of current techniques. In laboratory experiments, GPT of $O$ dioica was independent of body size. At $15^{\circ} \mathrm{C}$, GPT (min) decreased with increasing food concentration ( $F C, \mu \mathrm{g} \mathrm{C}^{-1}$ ) when the prymnesophyte Isochrysis galbana $(4.5 \mu \mathrm{m}$ in size), the prasinophyte Tetraselmis suecica $(10 \mu \mathrm{m})$ or the chlorophyte Chlorella sp. $(3 \mu \mathrm{m})$ were used as food, according to the power function GPT $=29.4 \mathrm{FC}^{-0.245}$. There were no significant differences in GPT between algal types. The GPT of $O$. dioica exhibited a $Q_{10}$ of 0.687 over a temperature range of 10 to $20^{\circ} \mathrm{C}$, independent of food concentration. Since the interaction between food concentration and temperature was not significant, GPT can be estimated as GPT = $51.67 \mathrm{e}^{-00376 t} \mathrm{FC}^{-0245}$.
\end{abstract}

KEY WORDS: Appendicularian · Feeding · Gelatinous zooplankton · Oikopleura - Tunicate · Gut passage time $\cdot$ Gut pigment

\section{INTRODUCTION}

Current functional response models place special emphasis on the compensatory role played by digestive processes (Penry \& Jumars 1987, Willows 1992). According to these models gut passage time (GPT, min) should respond to variations in the amount and quality of food to maximize net energy gain. Thus, GPT is a variable of paramount importance in the ecophysiology of marine organisms. Moreover, grazing rates of pelagic filter feeders have been calculated according to the gut pigment technique by dividing an

\footnotetext{
•E-mail: aurrutia@s@si.cpd.uniovi.es
}

in situ measurement of gut pigment content by an in vitro estimate of GPT (Mackas \& Bohrer 1976, Madin \& Cetta 1984). However, the task of measunng GPT in pelagic filter feeders by non-intrusive methods remains an elusive problem, and most current techniques rely on some important assumptions. For instance, the gut clearance (Mackas \& Bohrer 1976) and gut filling (e.g. Head 1986) techniques assume that GPT is independent of ingestion rate. Exposure to filtered seawater in gut clearance experiments can be avoided by using surrogate particles which do not interfere with the pigment analysis and that are readily ingested (e.g, charcoal, Perissinotto \& Pakhomov 1996), but this clearly influences the quality of the food offered. Use of radiolabelled food circumvents most of 
these caveats, but is hardly applicable during research cruises (Arashkevich 1977). Coloured markers, followed in their passage through the gut, are of common use for the transparent salps (e.g. cornstarch or carmine died cells, Madin \& Cetta 1984), at the cost of severely changing the quality of the food. Fecal. pellet production experiments which involve measurement of the total amount of pigments egested do not have these shortcomings, but rely on careful assesment of pigment degradation in gut and fecal pellets (e.g. Dagg \& Walser 1987).

Appendicularians are not exempt from these methodological problems. Measurement of the GPT in these animals has relied on the use of marker particles (Acuña et al. 1994, Bochdansky et al. 1998, Acuña et al. 1999), which involves manipulation of food quality and/or concentration. Moreover, it is often hard to ascertain the precise moment at which the marker is being ingested, because the food has first to be concentrated in the external filter house. In addition, shipboard measurement of GPT in these delicate tunicates represents a technical challenge, necessitating the use of onboard cold rooms in which to conduct the observations, and of suspension systems to isolate the animals from the ship vibrations (Acuña et al. 1999). This has lead researchers to simply rely on literature values, obtained under unreported experimental conditions, to calculate grazing rates by the gut pigment technique (e.g. Landry et al. 1994 used a GPT of 8 min reported by Alldredge 1981 for Oikopleura dioica). Wellfounded predictive equations are thus much needed.

Oikopleurid appendicularians are transparent, nonmotile animals in which food transit through the digestive system proceeds by an orderly sequence of fecal pellet translocations. Thus, appendicularians are ideal animals for the observation of gut throughput dynamics. Here we make use of these characteristics to propose a non-intrusive, observational method to measure GPT in Oikopleura dioica, and use this method to determine the influence of body size, food concentration, food type and temperature on the GPT. Finally, we use this ecophysiological knowledge to build a predictive model for the GPT of $O$. dioica, which can be extrapolated to field conditions.

\section{MATERIALS AND METHODS}

Appendicularians and phytoplankton cultures. Oikopleura dioica were collected from surface waters at the El Musel harbour in Gijón (N Spain), using plastic buckets, and quickly brought to walk-in controlled temperature rooms (set at the specified temperature $\pm 0.5^{\circ} \mathrm{C}$ ). Appendicularian cultures were initiated by placing healthy, wild-captured individuals inside their filter houses in $5000 \mathrm{ml}$ glass jars filled with $30 \mu \mathrm{m}$ filtered seawater which was continuously agitated by means of an acrylic spiral paddle rotating at $10 \mathrm{rpm}$ (Fenaux \& Gorsky 1985). The animals were transferred to fresh, $30 \mu \mathrm{m}$ filtered seawater every $2 \mathrm{~d}$.

The unicellular prasinophyte Tetraselmis suecica (10 $\mu \mathrm{m}$ ESD, Equivalent Sphaerical Diameter), the

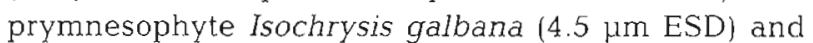
the unicelular chlorophyte Chlorella sp. ( $3 \mu \mathrm{m} \mathrm{ESD})$ were used as food for the experiments. Algae were cultured at $15^{\circ} \mathrm{C}$ in $1 \mathrm{l}$ bottles under $70 \mu$ Einstein white light, continuous aeration and 12:12 photoperiod. Only exponentially growing algae were used for the experiments.

Microscopic observation. Our data were collected by microscopic observation of individual Oikopleura dioica using an Olympus IMT-2 inverted microscope inside the controlled temperature room. To maintain the individual in a fixed position, a simple device was constructed using a plastic petri dish $(5.5 \mathrm{~cm}$ diameter, $1.5 \mathrm{~cm}$ height). A $1 \mathrm{~cm}$ wide hole was made through the lid of the dish, allowing insertion of a $1.5 \mathrm{~cm}$ long, cylindrical acrylic plastic tube that stood vertically on the petri dish. Both petri dish and plastic tube were filled with water at the target experimental temperature and food concentration. The individual was then transferred with a L-shaped, wide bore Pasteur pipette from the $5 \mathrm{l}$ glass jar to the observation device, along with a certain amount of preconditioning food suspension. The activity of the individual was recorded with a SONY SSC Color Video Camera fitted to the inverted microscope. The trunk length of the appendicularian, defined here as distance between the mouth and the posterior edge of the stomach, was measured on the video screen to the nearest $45 \mathrm{~nm}$

General experimental procedures. Algae for the experiments were centrifuged at $1500 \mathrm{RCF}$ (Relative Centrifugal Force) for 7 min and resuspended twice on GF/F filtered seawater, and the final concentration of the stock solution determined with a Coulter multisizer II fitted with a $70 \mu \mathrm{m}$ aperture tube. Organic carbon content of phytoplankton was estimated from cell volume using equations of Strathmann (1967). Because an appendicularian house lasts ca $4 \mathrm{~h}$, experimental individuals were allowed to precondition in $5 \mathrm{I}$ of $\mathrm{GF} / \mathrm{F}$ filtered seawater supplemented with algae up to the specified concentration for $4 \mathrm{~h}$, to ensure that they had enough time to expand a new filter house prior to observation. We did not use replicate conditioning jars because preliminary experiments showed no significant effects of this random factor on GPT (data not shown). Prior to ANOVA and regression analyses, data were examined for homogeneity of variance. Tests of linearity were conducted prior to regression analysis with replication. 


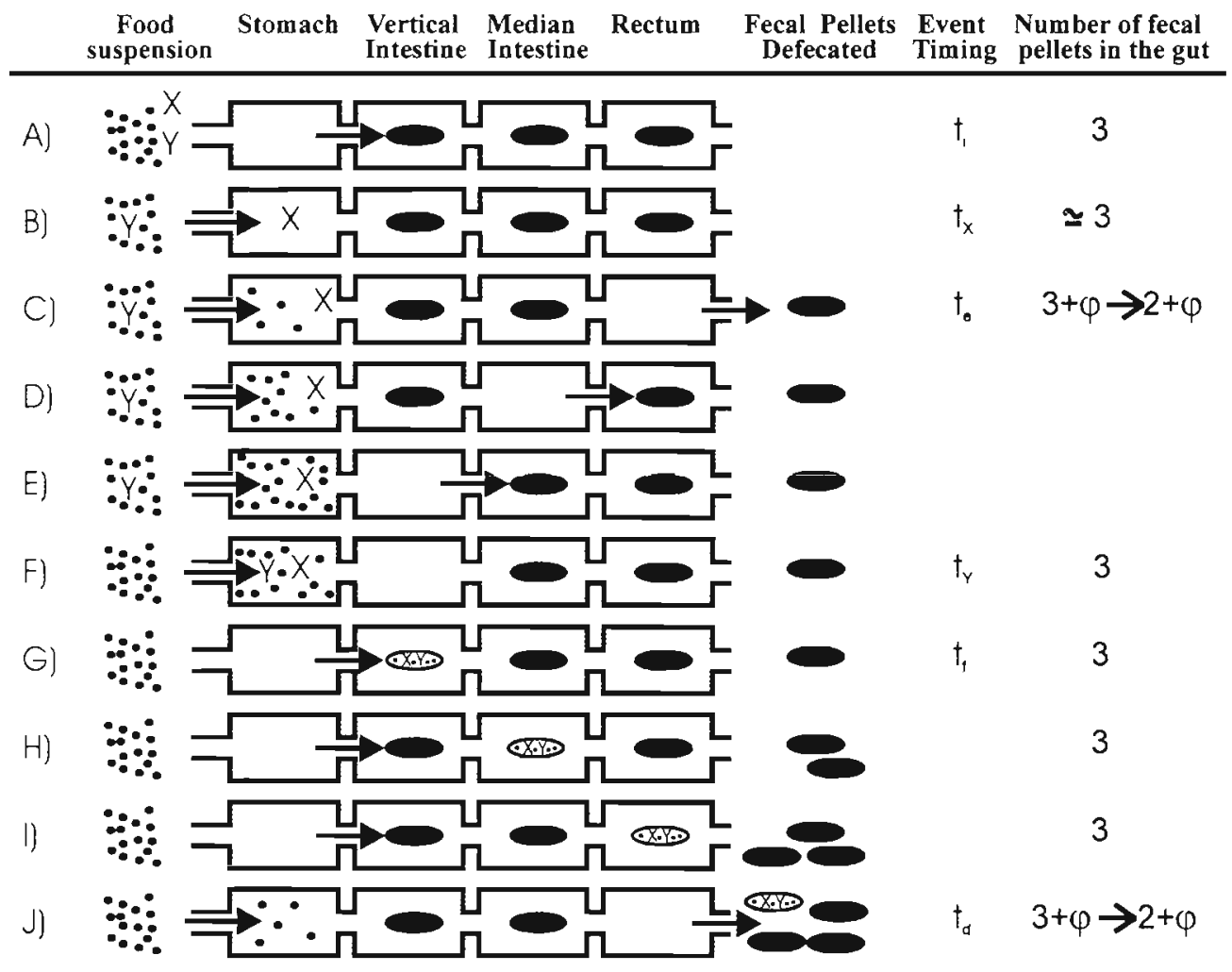

Fig. 2. Oikopleura dioica. Schematic representation of the chain of events (arrows) which takes place within the digestive system from incorporation of a particle into a fecal pellet to defecation. Events whose timing is pertinent to our measurements are assigned a time code under the heading 'event timing' (see 'Results'); gut contents (fecal pellets) are indicated under the heading 'Number of fecal pellets in the gut' (see 'Results' and Fig. 4). (A) Translocation of a newly formed fecal pellet from the stomach to the vertical intestine. Two other pellets are present in the median intestine and the rectum (this corresponds to Fig. 1B). (B) A new fecal pellet starts to form in the stomach. The first particle incorporated to that pellet is labelled X. (C) The fecal pellet in the rectum is defecated. More particles, labelled as dots, are incorporated into the fecal pellet which is being formed in the stomach (this corresponds to Fig. 1C). Gut content passes from $3+\varphi$ to $2+\varphi$ pellets, where $\varphi$ is the content of the stomach (in fecal pellets) during this moment. (D) The fecal pellet in the median intestine is translocated to the rectum. More particles enter in the stomach (this corresponds to Fig. 1D). (E) The fecal pellet in the vertical intestine is translocated to the median intestine. More particles accumulate in the stomach. (F) The last particle (Y) is incorporated into the fecal pellet which is being formed in the stomach. (G) The newly formed fecal pellet is translocated from the stomach to the vertical intestine. This phase of the cycle is the same as (A) above. $(\mathrm{H})$ Composite of several consecutive steps. The cycle starts over again and ends in a similar configuration as in (A \& G) above, but with the fecal pellet with particles labelled $X$ and $Y$ located in the median intestine. (I) Same as (A, $\mathrm{G}, \mathrm{H}$ ) above, but with the labelled fecal pellet located in the rectum. (J) Same as (C) above, but the labelled fecal pellet has just been defecated

been incorporated into a fecal pellet right at the beginning of its formation (i.e, particle $\mathrm{X}$ in Fig. 2B, incorporated at time $t_{x}$ ) spends more time within the digestive system than a particle incorporated at the end of the formation of the fecal pellet (particle $\mathrm{Y}$ in Fig. 2F, incorporated at time $t_{\gamma}$ ). Thus we must look for a collective or average time of incorporation representative of the population of particles belonging to this fecal pellet, rather than considering the time of incorporation of any individual particle. Given a constant rate of stomach filling, the population, average time of incorporation to the fecal pellet is the arithmetic mean of $t_{x}$ and $t_{y}$ or

$$
\frac{t_{\mathrm{y}}+t_{\mathrm{x}}}{2}
$$

However, we observe fecal pellets and their translocations, not individual particles, so it is impossible to measure $t_{\mathrm{y}}$ and $t_{\mathrm{x}}$. Because the animal has continuous feeding and it does not interrupt secretion of the pharyngeal filter, which keeps flowing along the esophagus while the pellet is translocated (authors' pers. obs.), incorporation of the first particle into the fecal pellet (particle $X$ at time $t_{\mathrm{x}}$, Fig. 2B) and translocation of the preceding fecal pellet from the stomach into the vertical intestine (at time $t_{\mathrm{i}}$, Fig. 2A) are consecutive events, and $t_{\mathrm{x}}=t_{1}$. Similarly, incorporation of the last particle to the fecal pellet (particle $Y$ at time $t_{y}$. Fig. 2F) and translocation of this fecal pellet into the vertical intestine (at time $t_{6}$, Fig. $2 \mathrm{G}$ ) are consecutive 


\section{RESULTS}

\section{Measurement of gut passage time in Oikopleura dioica}

The gut of Oikopleura dioica consists of a bilobate stomach, a vertical intestine, a median intestine and a rectum (Fig. 1, Fenaux 1989). In a healthy individual inside its filter house, it is possible to see 2 (Fig. 1C,D) or 3 (Fig. 1B) pellets distributed among the vertical and median intestines and the rectum, plus a variable amount of food in the stomach (no food in Fig. 1B, some food in Fig. $1 \mathrm{C}$ and an almost fully formed fecal pellet in Fig. 1D). These different pellet configurations can only be understood by analysing the dynamics of food flow within the digestive system of $O$. dioica (Fig. 2). Particles captured in the pharyngeal filter enter the stomach continuously and are progressively compacted into a fecal pellet (Fig. 2B-F). Once formed, this fecal pellet passes into the vertical intestine (Figs. $1 B$ \& $2 \mathrm{G}$ ) and is then sequentially translocated to the median intestine and to the rectum \{Fig. $2 \mathrm{H}, \mathrm{I}$ ) before its defecation (Fig. 2J). The movement of a fecal pellet through the digestive system is accompanied by a sequence of fecal pellet translocations in the preceding fecal pellets, which leave room for the following pellets (see Figs. 1 \& 2). Under constant ambient conditions, this sequence is extremely precise, repetitive, and can be accurately timed. It is possible to take advantage of this precise timing to develop an observational measurement of the GPT

To measure the GPT of a particle we need a precise timing of the moment of ingestion and defecation of that particle. All particles in a fecal pellet are defecated simultaneously, and this event can be visualiy timed (i.e. Fig 1C, $t_{\mathrm{d}}$ in Fig. 2J). However, not all particles are ingested and incorporated into a forming fecal pellet at the same moment. A food particle that has
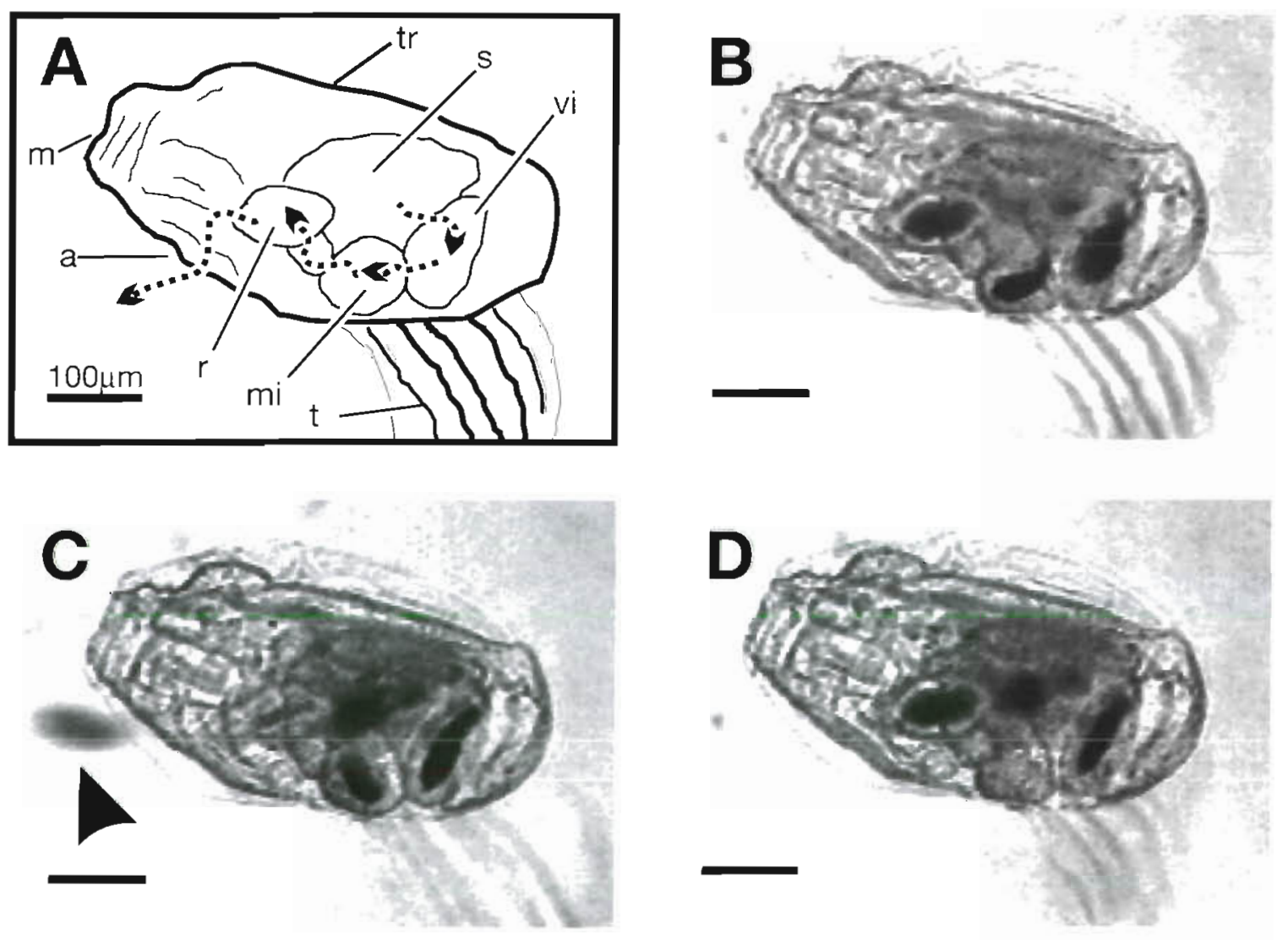

Fig. 1 Oikopleura dioica. (A) Sketch of the trunk showing the different parts of the digestive system, based on Fig. 1B. a: anus; m: mouth; mi: median intestine; r: rectum; s: stomach; t: tail; tr: trunk, and vi: vertical intestine. Dashed arrows indicate the path followed by the fecal pellets through the digestive system. (B,C,D) Micrographies of the trunk of an actively filtering individual Individuals for these video-images have been incubated in a dilute suspension of sepia ink; the ink is then ingested and incorporated into the fecal pellets, which then appear heavily stained. The arrowhead in (C) signals a fecal pellet which has just been defecated. (To understand how these different configurations are temporally linked, see Fig. 2 and 'Results'.) These configurations correspond to schematic representations in Fig. 2A, G, H,I for (B), Fig. 2C,J for (C) and Fig. 2I) for (D) 


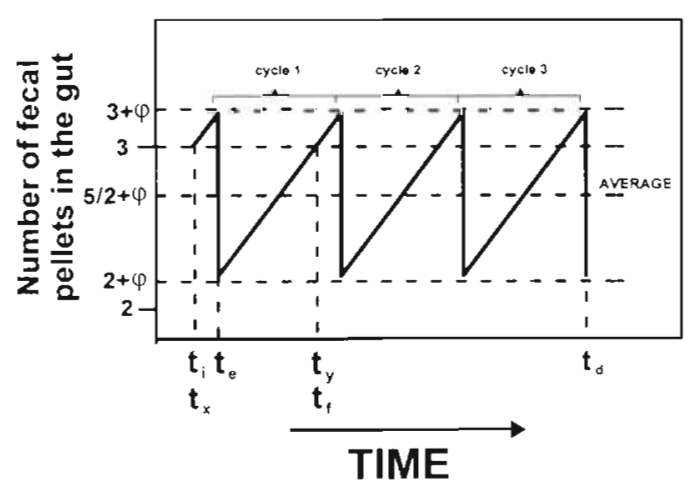

Fig. 4. Oikopleura dioica. Temporal changes in the number of fecal pellets within the gut according to the diagram in Fig. 2 and assuming a linear increase in the stomach content

From our observations, and according to Eq. (3), the average value of $\varphi$ was $0.368 \pm 0.013$ (mean $\pm \mathrm{SD}, \mathrm{n}=$ 43). When inserted into Eq. (4), this value of $\varphi$ yields an average number of fecal pellets of $2.868 \pm 0.013$, which is close to the slope of the regression of GPT versus DI $\left(2.878_{i}\right.$ Eq. 2$)$ and indicates to us that we are right in accepting a 0 intercept for that equation.

\section{Factors affecting GPT}

\section{Effect of body size}

Expt 1A. This experiment was designed to test for differences in GPT associated with body size through a linear regression approach. GPT of 24 individuals of differing trunk length were observed at a concentration of $120 \mathrm{\mu g} \mathrm{Cl}^{-1}$ of Isochrysis galbana and $15^{\circ} \mathrm{C}$ temperature. No significant effect of trunk length on GPT was detected within a trunk length range from 0.25 to $1.22 \mathrm{~mm}$ (Fig. 5). However, the low power of the regression (1- $\beta_{\alpha c=0.05}=0.3974$ ) and the low $p$-value $\left(F_{1,23}=3.307, \mathrm{p}=0.082 ; \mathrm{n}=25, \mathrm{r}^{2}=0.126\right.$; Fig. 5$)$ suggest that we could be incurring a Type II error, which warrants further confirmation of this result (see next experiment).

Expt 1B. This experiment was designed to test for differences in GPT due to body size through an ANOVA approach. The idea was to incubate animals belonging to 2 cohorts of differing body size to test for differences in GPT between cohorts (i.e. between sizes). We used large $(0.903 \pm 0.034 \mathrm{~mm}$ trunk length $\pm \mathrm{SD}, \mathrm{n}=15)$ and small $(0.242 \pm 0.061 \mathrm{~mm} ; n=15)$ individuals for the experiments, which were performed at $15^{\circ} \mathrm{C}$ and a concentration of $100 \mu \mathrm{g} \mathrm{Cl}^{-1}$ of either Isochrysis galbana, Tetraselmis suecica or Chlorella sp. Thus, we used a 2-way orthogonal design with 2 levels of body size (large/small) and 3 levels of food type ( 3 algal species).

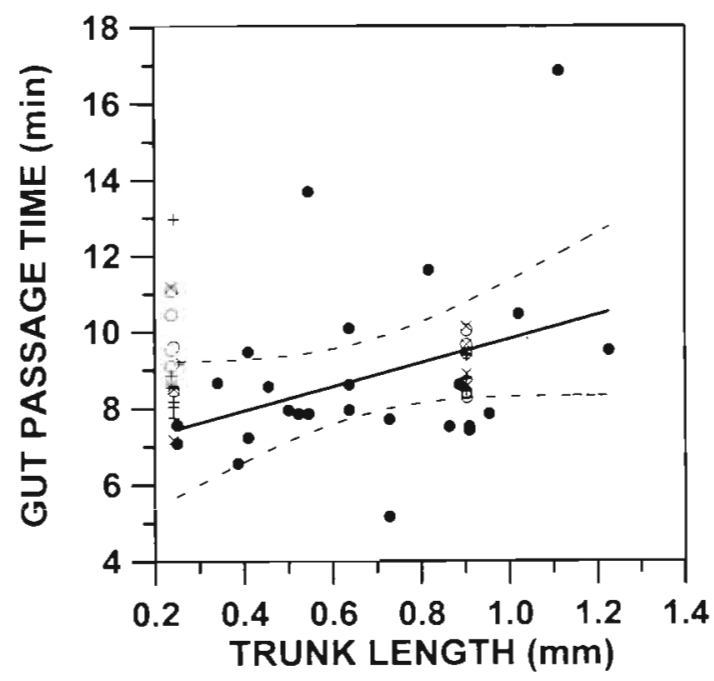

Fig. 5. Oikopleura dioica. Plot of GPT (min) versus trunk length $(\mathrm{mm})$. (•) Data from Expt 1A. (-) least square regression of GPT versus trunk length for that experiment

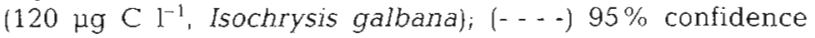
intervals. The slope of the linear regression was not significantly different from zero $\left(\mathrm{r}^{2}=0.13 ; \mathrm{n}=43 ; F_{1,23}=3.31, \mathrm{p}=\right.$ 0.082 ; power of the regression is $\left.1-\beta_{\alpha}=0.05=0.40\right) .(0),(x)$ and $(+)$ data from Expt $1 \mathrm{~B}$ (see Table 1 and 'Results'). In that experiment, individuals belonging to 2 distinct appendicularian size classes were incubated at $100 \mu \mathrm{g} \mathrm{C} \mathrm{l^{-1 }}$ of $I$. galbana $(0)$, Chlorella sp. $(x)$ and Tetraselmis suecica $(+)$

This design allowed us to test for the existence of interactions between trunk length and algal type, indicative of differences in the ability to capture or ingest phytoplankton of different size or taxonomic group between the small and large animals. There were neither significant effects of trunk length $(p=0.71)$ or food source $(p=0.66)$, nor significant interaction between these 2 factors ( $p=0.70$, Table 1, Fig. 5).

\section{Effect of food concentration and algal species}

Expt $2 \mathrm{~A}, \mathrm{~B}$ and $\mathrm{C}$. GPT was measured at 5 different food concentrations of Isochrysis galbana, Tetraselmis suecica and Chlorella sp. in 3 separate experiments at $15^{\circ} \mathrm{C}$ (Expt $2 \mathrm{~A}, \mathrm{~B}$ and $\mathrm{C}$, respectively) to test the effect of food concentration and algal type on GPT. The relationship between GPT and food concentration for each type of alga was fitted to a power relationship (Fig. 6A,B,C). We chose a power function to fit GPT versus food concentration, because this model has been previously used for similar data in copepods (Dagg \& Walser 1987). Estimated slopes for the regression lines were significantly different from zero for all 3 algae ( $p<0.01$, Fig. 6), which indicated that food concentration had a significant effect on GPT. 
events and $t_{\mathrm{y}}=t_{\mathrm{f}}$. This implies that the average time of incorporation to the fecal pellet will be given by

$$
\frac{t_{\mathrm{f}}+t_{\mathrm{i}}}{2}
$$

It follows that the average gut passage time (GPT) can be calculated as the difference between the time of the defecation of a fecal pellet and the average time of incorporation of particles to that pellet, that is

$$
\mathrm{GPT}=t_{\mathrm{d}} \frac{t_{\mathrm{f}}+t_{\mathrm{i}}}{2}
$$

\section{Estimation of the GPT from the defecation interval}

Measurement of GPT as explained above involves observing individual animals from start of formation of a fecal pellet $\left(t_{i}\right.$, Fig. $\left.2 \mathrm{~A}\right)$ to defecation $\left(t_{d}\right.$, Fig. $\left.2 J\right)$. Even at the fastest GPT achievable, observations by this technique take more than 6 min for each individual, so we looked for alternative, less time-consuming ways of measuring GPT.

Each complete observation of GPT involved observing the defecation of 4 fecal pellets, and consequently we also could measure 3 time intervals between consecutive fecal pellets (see Fig. 2). Thus we looked at the time interval between fecal pellets (DI, min fecal pellet $^{-1}$ ) as a proxy to estimate GPT, and compared the average of these 3 DI against GPT for a total of 43 individuals under contrasting ambient conditions (Fig. 3). Due to the existence of natural mutual variability in the observations, a Geometrical Mean Regression (GMR, Ricker 1984) was used instead of an ordinary least squares regression. There is a robust, linear relationship between GPT (min) and average DI (min fecal pellet $^{-1}$ ) with 0 intercept (Fig. 3), which indicates that DI is a constant proportion of GPT, i.e.

$$
\mathrm{GPT}=2.878 \mathrm{DI}
$$

Since no significant differences were found between consecutive measurements of DI (ANOVA for repeated measures, $F_{2,84}=1.902, \mathrm{n}=43, \mathrm{p}=0.15$, power of test $_{\alpha=0.05}=0.615,3$ consecutive measurements for each individual) only 1 measurement of DI was recorded per individual thereafter. The corresponding GPT was then calculated according to Eq. (2), which allowed us to reduce manipulation of animals and increase the number of observations per experiment.

\section{Number of fecal pellets inside the gut of Oikopleura dioica}

The coefficient 2.878 in the right hand term of Eq. (2) represents the average number of fecal pellets in the

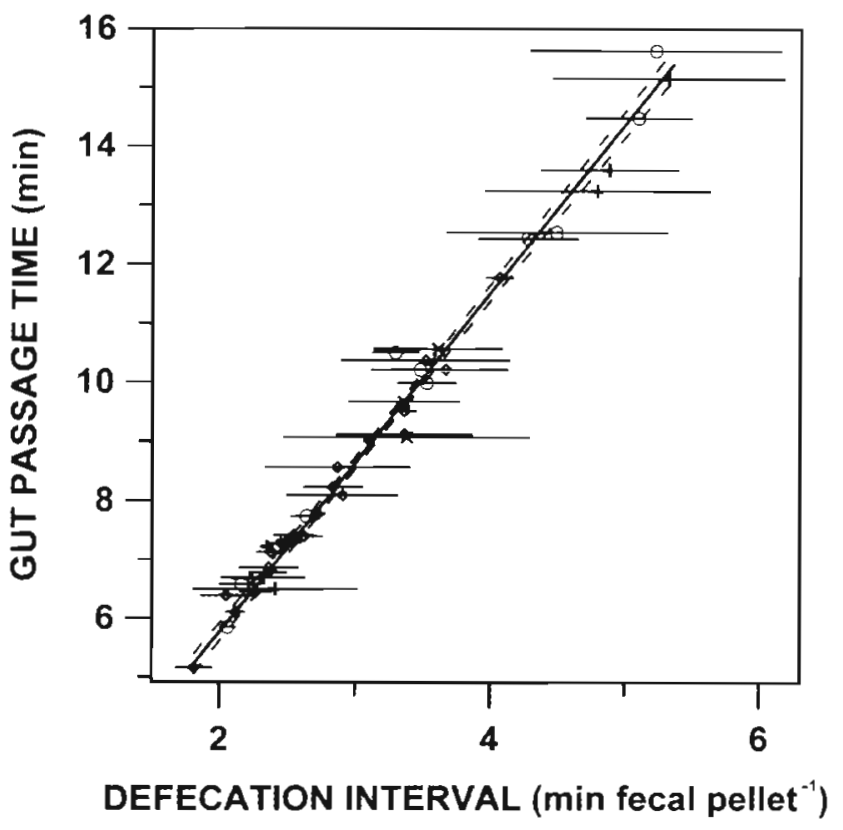

Fig. 3. Oikopleura dioica. Plot of GPT (min) versus DI (min fecal pellet ${ }^{-1}$ ) for animals incubated at a range of food concentrations and food types and $15 \pm 0.5^{\circ} \mathrm{C},(0),(0)$ and $(+)$ observations conducted on animals incubated at food concentrations of 60,120 and $240 \mu \mathrm{g} \mathrm{C} \mathrm{l}^{-1}$ of Isochrysis galbana, respectively; $(x)$ observations conducted on animals incubated on unfiltered seawater. Horizontal solid lines: SD. Solid line: Geometrical Mean Regression; dashed lines: 95\% confidence intervals. GPT (min) can be estimated from DI (min fecal pellet $^{-1}$ ) according to the regression equation GPT $=$ $(2.878 \pm 0.015) \mathrm{DI}\left(\right.$ mean $\pm \mathrm{SE}_{i} \mathrm{x}^{2}=0.9825, \mathrm{n}=43, F_{1,42}=$ 35793.87, $\mathrm{p}<0.001$ ). The regression intercept was forced through zero because the intercept of an ordinary regressionwas not significantly different from zero $(t=1.618, \mathrm{p}=0.11)$

gut (i.e. GPT/DI). In fact, it is possible to obtain an independent measurement for the average number of fecal pellets (AFP, fecal pellet) inside the gut of an individual Oikopleura dioica by timing characteristic events in the fecal pellet formation and circulation. According to this analysis (Fig. 2) the number of fecal pellets inside the gut of $O$. dioica experiences oscillations between a minimum value of $2+\varphi$ and a maximum value of $3+\varphi$, where $\varphi$ (fecal pellets) is the stomach content when a fecal pellet is defecated (Fig. 4), and can be calculated as:

$$
\varphi=\frac{t_{e}-t_{\mathrm{i}}}{t_{\mathrm{f}}-t_{\mathrm{i}}}
$$

Since this cycle is repeated continuously, the AFP within each cycle will also be the time-integrated AFP in the gut of Oikopleura dioica, which should therefore be calculated as

$$
\mathrm{AFP}=\frac{5}{2}+\varphi
$$




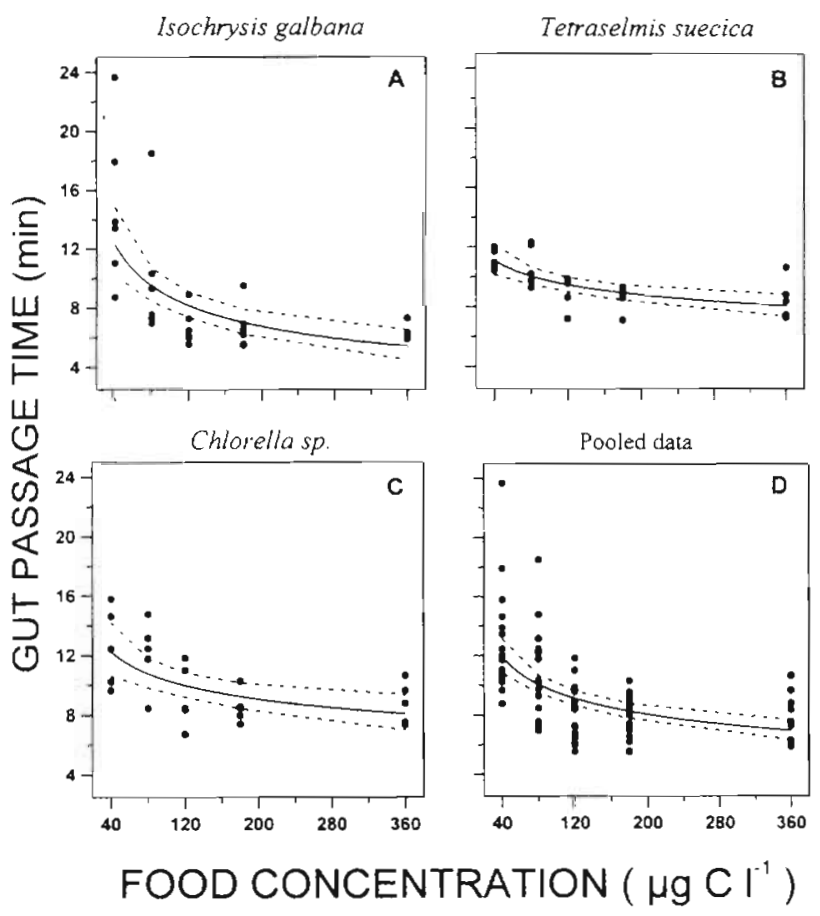

Fig. 6. Oikopleura dioica. Plot of GPT (min) versus food concentration ( $\left.\mu \mathrm{g} \mathrm{C}^{-1}\right)$ for individuals incubated in (A) Isochrysis galbana; (B) Tetraselmis suecica; (C) Chlorella sp. and (D) for all data pooled. (-) Least square regressions for data fitted to a power model; (- - -) $95 \%$ confidence intervals. Power regression equations between GPT (min) and food concentration ( $\left.\mathrm{FC}, \mu \mathrm{g} \mathrm{C} \mathrm{l^{-1 }}\right)$ are: $\mathrm{GPT}=(48.95 \pm 16.33)$ $F C^{(-0.374=0.069)}\left( \pm \mathrm{SE}, \mathrm{r}^{2}=0.51, \mathrm{n}=30, F_{1.28}=29.52, \mathrm{p}<0.001\right)$ for $I$. galbana; $\mathrm{GPT}=(19.038 \pm 3.057) \mathrm{FC}^{(-0.146 * 0.033)}\left( \pm \mathrm{SE}, \mathrm{r}^{2}=\right.$ $0.46, \mathrm{n}=25, F_{1.23}=19.47, \mathrm{p}<0.001$ ) for $T$. suecica; $\mathrm{GPT}=$ $\left.(24.61 \pm 6.3) \mathrm{FC}^{i-0.188}+0.052\right)\left( \pm \mathrm{SE}, \mathrm{I}^{2}=0.36, \mathrm{n}=25, F_{1,23}=\right.$ $12.72, \mathrm{p}=0.0016)$ for Chlorella sp. and GPT $=(29.4 \pm 5.1)$ $\mathrm{FC}^{(-0.245 \pm 0.036)}\left( \pm \mathrm{SE}_{\mathrm{i}} \mathrm{r}^{2}=0.37, \mathrm{n}=80, F_{1,78}=45.97, \mathrm{p}<0.001\right.$, Kolmogorov-Smirnov test for normality of residuals, $Z=1.14$, $p=0.15)$ for the pooled data

concentration. Since the parameter $c$ in Eq. (6) does not depend on food concentration, we can combine Eq. (5), which describes the relationship between GPT and food concentration $\left(\mathrm{FC}_{,} \mu \mathrm{g} \mathrm{Cl}^{-1}\right)$ as measured at $15^{\circ} \mathrm{C}$, with Eq. (6) for a temperature of $15^{\circ} \mathrm{C}$ and solve for $k$ to arrive at:

$$
k=51.67 \mathrm{FC}^{-0.245}
$$

If $k$ is substituted in Eq. (6) by the right-hand term of Eq. (7), Eq. (6) becomes:

$$
\mathrm{GPT}=51.67 \mathrm{FC}^{-0.245} \mathrm{e}^{-00376 t}
$$

This equation can be used to estimate gut passage time from knowledge of the concentration of available food ( $\mathrm{FC}$, in $\left.\mu \mathrm{g} \mathrm{Cl}^{-1}\right)$ and ambient temperature $\left(t,{ }^{\circ} \mathrm{C}\right)$.

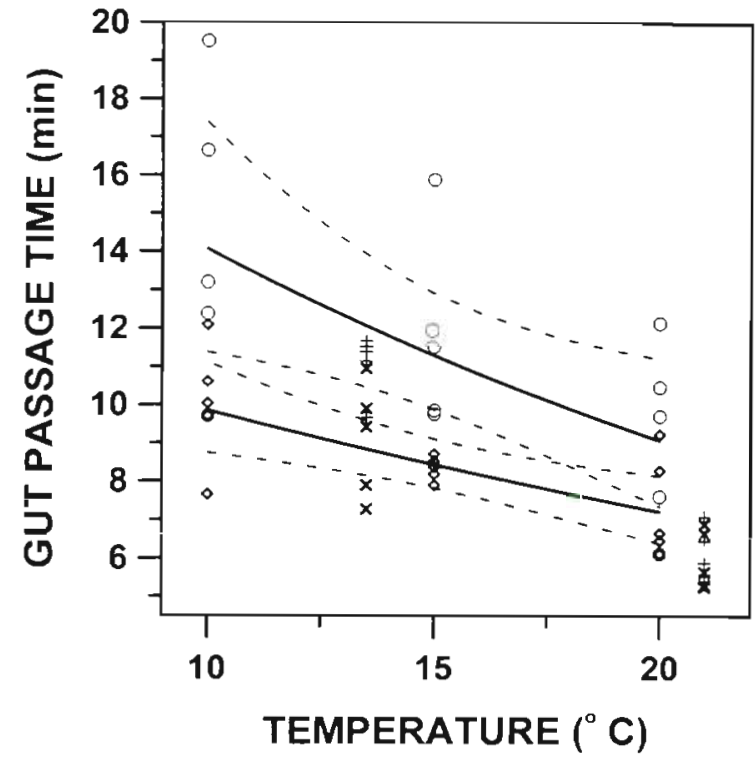

Fig. 7. Oikopleura dioica. Plot of GPT (min) versus temperature $\left(t,{ }^{\circ} \mathrm{C}\right)$. Data at 10,15 and $20^{\circ} \mathrm{C}$ correspond to Expt $3 \mathrm{~A}$, in which the animals were incubated at concentrations of $30(0)$ and $250(\diamond) \mu \mathrm{g} \mathrm{Cl}^{-1}$ of Isochrysis galbana (see Table 1 and 'Results'). ( - Least squares fits to an exponential model for data at $30 \mathrm{\mu g} \mathrm{C} \mathrm{l}^{-1}$ (upper solid line, GPT $=3.08 \pm 0.24$ $\left.\mathrm{e}^{-0.044 \pm 0.015 \mathrm{t}}, \pm \mathrm{SE}, \mathrm{r}^{2}=0.34, \mathrm{n}=15, F_{1.13}=8.35, \mathrm{p}=0.013\right)$ and $250 \mu \mathrm{g} \mathrm{Cl}^{-1}$ (lower solid line, GPT $=2.60 \pm 0.13 \mathrm{e}^{-00313 \pm 00087 t}$ $\left.\mathrm{r}^{2}=0.50, \mathrm{n}=15, F_{1.13}=13.02, \mathrm{p}=0.003\right) .(---) 95 \%$ confidence intervals for the regressions. Data at 13.5 and $21^{\circ} \mathrm{C}$ correspond to Expt 3B, in which the individuals were incubated at a concentration of $60 \mu \mathrm{g} \mathrm{Cl}^{-1}$ of $I$. galbana (+) and Chlorella sp. $(\times)$, see Table 1 and 'Results'

\section{DISCUSSION}

\section{The gut of Oikopleura dioica: a chemical reactor perspective}

None of the chemical reactor types described by Penry \& Jumars (1987) exactly describes the gut of Oikopleura dioica. This is important, since the choice of reactor determines the digestion strategy. While the intestine-rectum of $O$. dioica functions like a plug flow reactor (i.e. a conveyor belt carrying fecal pellets, Fig. 2) the stomach functions in a way such that it exhibits characteristics of both a batch-reactor (i.e. it has pulsed outflow) and a continuous-flow, stirred tank reactor (i.e. it has continuous inflow, Fig, 2). Most planktonic filter feeders are similar in their continuous ingestion and discrete defecation, with the main differences arising in the way the fecal pellets are compacted. For example, only a proportion of the foregut contents are packed in a fecal pellet in copepods (Gauld 1957), while appendicularians pack the whole foregut (i.e. the stomach) content, which is thus emptied each time a fecal pellet is formed (Fig. 1B). 
Next we compared the results of these 3 experiments to test for effects due to food type. Because variances in. GPT between the 3 experiments were non homogeneous (Bartlett Chi-square test $B_{14}=26.497, p=0.02$ ), we used a Rank Analysis of Covariance (Quade 1967. cited in Huitema 1980). No significant effect of food type on GPT was detected $\left(F_{2,77}=2.68, \mathrm{p}=0.075\right.$; Table 1), which is in agreement with the result obtained in Expt 1B, above. Therefore, we pooled data from all 3 algae to estimate a power regression of GPT versus food concentration, which explained $37 \%$ of the total variance in GPT (Fig. 6D). According to this equation, gut passage time at $15^{\circ} \mathrm{C}\left(\mathrm{GPT}_{15^{\circ} \mathrm{C}}\right.$, min) can be

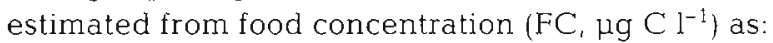

$$
\mathrm{GPT}_{15^{\circ} \mathrm{C}}=29.4 \mathrm{FC}^{-0.245}
$$

\section{Effect of temperature}

Expt 3A. To test the effect of temperature, we observed the GPT of individuals raised from egg to age

Table 1 Summary of ANOVA and ANCOVA experiments, including the sum of squares (SS), degrees of freedom (df), mean square (MS), $F$ and p values. In all cases the dependent variable is gut passage time (GPT), except in Expt 3A, where the dependent variable was the natural logarithm of GPT

Independent variables (ind. vars.) are indicated in each experiment

\begin{tabular}{|c|c|c|c|c|c|}
\hline Source of variation & SS & df & MS & F & $\mathrm{p}$ \\
\hline \multicolumn{6}{|c|}{ Expt 1B (2-way ANOVA: ind. vars. = algal species \& trunk length) } \\
\hline Main effects & 2.62 & 5 & 0.52 & 0.34 & 0.81 \\
\hline Algal species & 1.29 & 2 & 0.65 & 0.42 & 0.66 \\
\hline Trunk length & 0.22 & 1 & 0.22 & 0.14 & 0.71 \\
\hline Algal species $x$ trunk length & 1.10 & 2 & 0.55 & 0.36 & 0.70 \\
\hline Model & 2.62 & 5 & 0.52 & 0.34 & 0.88 \\
\hline Error & 37.09 & 24 & 1.54 & - & - \\
\hline Total residual & 39.71 & 29 & 1.37 & - & - \\
\hline \multicolumn{6}{|c|}{$\begin{array}{l}\text { Expt } 2 \text { (rank ANCOVA: covariate = food concentration; ind. var. = } \\
\text { algal species) }\end{array}$} \\
\hline Algal species & 5952 & 2 & 2976 & 2.68 & 0.075 \\
\hline Error & 85579 & 77 & 1111 & - & - \\
\hline Total residual & 91531 & 79 & 1158 & - & - \\
\hline \multicolumn{6}{|c|}{$\begin{array}{l}\text { Expt } 3 \mathrm{~A} \text { (1-way ANCOVA: covariate = temperature; ind var. }= \\
\text { food concentration) }\end{array}$} \\
\hline Temperature & 0.70 & 1 & 0.71 & 18.81 & $<0.001$ \\
\hline Food Concentration & 0.64 & 1 & 0.64 & 17.15 & $<0.001$ \\
\hline Model & 1.35 & 2 & 0.67 & 17.98 & $<0.001$ \\
\hline Error & 1.01 & 27 & 0.04 & - & - \\
\hline Total residual & 2.36 & 29 & 0.08 & - & - \\
\hline \multicolumn{6}{|c|}{ Expt 3B (2-way ANOVA: ind. vars. = algal species \& temperature $)$} \\
\hline Main effects & 1.37 & 2 & 0.69 & 43.69 & $<0.001$ \\
\hline Algal species & 0.07 & 1 & 0.07 & 4.17 & 0.058 \\
\hline Termperature & 1.31 & 1 & 1.31 & 83.2 & $<0.001$ \\
\hline Algal species $\times$ temperature & 0.04 & 1 & 0.04 & 2.72 & 0.119 \\
\hline Model & 1.42 & 3 & 0.47 & 30.03 & $<0.001$ \\
\hline Error & 0.25 & 16 & 0.02 & - & - \\
\hline Total residual & 1.67 & 19 & 0.09 & - & - \\
\hline
\end{tabular}

$4 \mathrm{~d}$ at 10,15 and $20 \pm 0.5^{\circ} \mathrm{C}$ and at 30 and $250 \mu \mathrm{g} \mathrm{C} \mathrm{l^{-1 }}$ of Isochrysis galbana. The idea was to estimate a $Q_{10}$ value for the GPT, to determine if this estimated $Q_{10}$ was different for the 2 algal concentrations and, if not different, to calculate a common $Q_{10}$ valid for both concentrations. GPT(min) versus temperature $\left(t,{ }^{\circ} \mathrm{C}\right)$ data for each concentration were fitted to an exponential model following the Arrhenius equation, i.e. GPT = $a e^{\mathrm{c} t}$, or $\ln \mathrm{GPT}=\ln a+c t$ (Fig. 7). Regression slopes (i.e. $c$ in the Arrhenius equation) were not significantly different for the different food concentrations (test of parallelism: $F_{1,26}=0.52, p=0.48$ ), and the estimate for the common regression slope, which was significantly different from zero $\left(t_{27}=-4.337, \mathrm{p}<0.001\right)$, is $\mathrm{c}=$ $-0.0376 \pm 0.0087$ (mean $\pm \mathrm{SE}$ ). However, there were significant differences in adjusted means among food concentrations (ANCOVA, $F_{1,27}=18.81, p<0.001$; Table 1), which is in agreement with Expt $2 \mathrm{~A}, \mathrm{~B}$ and $\mathrm{C}$ (Fig. 6). According to this result, GPT (min) can be estimated from knowledge of the environmental temperature $\left(t,{ }^{\circ} \mathrm{C}\right)$ as

$$
\mathrm{GPT}=k \mathrm{e}^{-0.0376 !}
$$

where $k$ is a coefficient which depends on the food concentration. A common slope estimate of -0.0376 corresponds to a $Q_{10}$ of 0.687 .

Expt 3B. We wanted to know if different algal types might influence the response of GPT to temperature (i.e. the $Q_{10}$ ). Here we used a simpler design than that in Expt $3 \mathrm{~A}$, and incubated individuals for measurement of GPT at 2 temperatures $\left(13.5 \pm 0.5\right.$ and $\left.21^{\circ} \mathrm{C} \pm 0.5\right)$

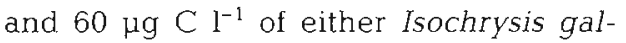
bana or Chlorella sp. No significant interaction was found between temperature and algal species used as food $\left(F_{\mathrm{L}, 16}\right.$ $=2.71592, \mathrm{p}=0.119,1-\beta_{\alpha=0.05}=0.34$, Table 1), which confirms that the $Q_{10}$ value of 0.687 can be used for different types of food.

\section{Prediction of GPT}

According to the experiments above only food concentration and temperature significantly influenced GPT, and Expt 3A confirmed that there was no interaction between these 2 factors. Here we use this knowledge to derive a simple equation for predicting GPT from ambient temperature and food concentration. Eq. (6) requires knowledge of the exact value of a parameter $k$, which is dependent on food 
Although we are far from explaining these differences, they clearly suggest that evolution has progressed well beyond chemical engineering in the design of optimal digestive strategies.

\section{Measurement of GPT in Oikopleura dioica: implications for the estimation of grazing rates by the gut pigment technique}

One of the characteristics of batch reactors with continuous filling is that different food particles have different gut residence times, defined as the time an individual element spends in the gut. Penry \& Jumars (1987) defined gut passage time as the mean gut residence time, and recommended the use of gut passage time as a measure of gut throughput time (i.e the time for 1 gut volume of food to be processed). Our newly developed method for the measurement of GPT in Oikopleura dioica is perfectly consistent with this definition. Previous determinations of GPT in appendicularians have relied on the use of marker particles (Alldredge 1981, Acuña et al. 1994, Bochdanksy et al. 1998, Bochdansky \& Deibel 1999, Acuña et al. 1999), and are therefore gut residence time measurements. Moreover, because our method is based on timing the defecation of 2 consecutive fecal pellets, it does not require manipulation of food quality or quantity. The existence of a relationship between defecation interval and gut passage time in appendicularians (Eq. 2) has been already pointed out by Bochdansky \& Deibel (1999), who proposed that, for continuously feeding appendicularians, GPT should equal the amount of food in the gut (in fecal pellets) times the average defecation interval. Their estimates for GPT, after assuming a value of 3 as the aproximate number of fecal pellets in the gut of Oikopleura vanhoeffeni, were very close to measurements using diatoms and cornstarch as markers. Recent shipboard observations conducted in the North Water Polynya have confirmed that our observational method is also applicable to this species (Acuña et al. unpubl.)

This method could also be a simple, accurate, and non-intrusive substitute for current gut clearance (Mackas \& Bohrer 1976), gut filling (Head 1986), or radiotracer methods (Arashkevich 1977) for determining GPT in copepods. In a revision of data from Arashkevich (1977) and Arashkevich \& Tseytlin (1978), Baars \& Oosterhuis (1985) recommend the estimation of gut passage time from the time interval between egestion of consecutive fecal pellets, after calculating an average of 2.9 pellets inside the gut for 4 copepod species. The use of gut clearance rate experiments to estimate gut passage time in marine copepods has been criticised because of the unlikely assumption that gut clearance rate is independent of ingestion (see Dam \& Peterson 1988 and references therein). Underestimation of grazing rates by the gut pigment technique when compared with other methods was explained by Peterson et al. (1990) as a consequence of using gut evacuation rate constants from evacuation experiments on filtered seawater as estimates of GPT. However, when GPT was estimated as twice the mean defecation interval, ingestion rates estimated by the gut pigment content technique were similar to values obtained by other techniques (Peterson et al. 1990).

Measurement of the time interval between consecutive fecal pellets is conceptually linked to the measurement of fecal pellet production rates (the inverse of the time interval between consecutive fecal pellets). This suggests a different approach for the measurement of GPT, based on recording the number of fecal pellets produced within a given time interval. Obviously this approach involves blind incubation rather than microscopical observation, and opens a simple avenue to conduct GPT measurements by incubation in thermo-insulated flasks onboard a ship, where availability of walk-in cold rooms may be limited. In a biogeochemical context, combination of these fecal pellet production rates with fecal pellet sinking velocities would allow the calculation of vertical pellet fluxes. Obviously these values would only represent maximum flux estimates, as they do not take into account the possibility of fecal pellet recycling or the existence of non-feeding periods when building new filter houses.

A further implication of our observations regards the variability of in situ gut pigment content measurements. We have found clear evidence that the number of fecal pellets within the gut of Oikopleura dioica oscillates between $3+\varphi$ and $2+\varphi$, with an average at

$$
\frac{5}{2}+\varphi
$$

(Fig. 4), being $\varphi=0.368$. This means that the gut content (i.e. the gut pigment content) systematically varies between $135 \%$ and $65 \%$ of the average gut content. This variation is due to the mechanism of digestion of $O$. dioica and is independent of other intrinsic (i.e. body size) or environmental (i.e. temperature, food concentration and source) factors. When we capture animals from the field for analysis of individual gut pigment contents we are taking snapshots of this oscillatory cycle; therefore these measurements are tied to a non-reducible variability that cannot be explained by regression on environmental variables or body size. In fact, regression models to predict the gut pigment contents of wild-captured oikopleurids 
explain only limited amounts of variance (Acuña 1999). We see no reason why the copepod gut should differ in regard to this intrinsic variability (see Fig $4 \mathrm{~A}$ \& B in Caparroy \& Carlotti 1996 for a theoretical example), although gut pigments of copepods are seldom analysed individually.

\section{Ecophysiology of GPT in Oikopleura dioica}

We detected a non significant effect of body size on GPT, through both a regression experiment involving an even distribution of sizes and an ANOVA experiment in which 2 different cohorts of different size were compared (Fig. 5, Table 1). GPT seems to be unrelated to body size in other appendicularian species (Bochdansky et al. 1998), in some salps (Madin \& Cetta 1984) and in copepods (Morales et al. 1990). This is interesting, because all effects of body size on ingestion rates are confined to gut contents, which greatly simplifies the task of building predictive models for GPT (i.e. Eq. 8)

As expected, GPT responded to temperature within the range of temperatures typical of surface waters off the Cantabrian coast $\left(10\right.$ to $20^{\circ} \mathrm{C}$. Acuña \& Anadón 1992). A $Q_{10}$ value of 0.687 for GPT implies that its inverse, the gut evacuation rate constant $\left(\mathrm{min}^{-1}\right)$, has a $Q_{10}$ of 1.46 . This is lower than $Q_{10}$ values for the gut evacuation rate constant for copepods (2.24, Dam \& Peterson 1988) which indicates that feeding rates of the eurithermal Oikopleura dioica are less sensitive to temperature. It is also lower than the $Q_{10}$ measured by Gorsky et al. (1987) for the respiration rate of $O$. dioica (1.96, after digitizing their Fig. 3 for calculation of a respiratory $Q_{10}$ for body size-corrected data over the temperature range of 15 to $24^{\circ} \mathrm{C}$ ). $A Q_{10}$ close to 1.4 is what should be expected for the respiration rates of poikilotherms (Peters 1983).

GPT also varied within the range of suspended organic carbon concentrations typical of the coastal waters where Oikopleura dioica is present (e.g. Alldredge 1981), which confirms that a reduced food supply is in part compensated by increased digestion times in accordance with current digestion theory (Willows 1992). Bochdansky et al. (1998) found a low, non significant effect of food concentration on gut passage time in the cold water Oikopleura vanhoeffeni, while Dagg \& Walser (1987) detected a clear response of GPT for the copepod Neocalanus plumchrus below a threshold chlorophyll concentration of $4 \mu \mathrm{gl}^{-1}$. Assuming a carbon:chlorophyll ratio of 40 , this would correspond to $160 \mu \mathrm{g} \mathrm{C} \mathrm{I}^{-1}$, which is close to our threshold at ca $120 \mu \mathrm{g} \mathrm{Cl}^{-1}$ (Fig. 6D).

Although we detected no significant effects of algal species on GPT, p values for these statistical tests were sufficiently close to 0.05 to take this result cautiously (Table 1; Expts 1B, 2 and 3B). Moreover, the curve of GPT versus food concentration for Isochrysis galbana had a markedly different aspect from that for Tetraselmis suecica and Chlorella sp., which is consistent with differences between feeding functional response curves of Oikopleura dioica when feeding on these same algal species (Acuña \& Kiefer in press). The effect was small, however, when compared with the effects of temperature and food concentration, but we are probably not sampling the full range of particle qualities that $O$. dioica encounters in nature. More research is clearly needed in this regard.

\section{Prediction of GPT in Oikopleura dioica}

We found no significant interactions between food concentration and temperature on the GPT of Oikopleura dioica (Table 1). This indicated to us that temperature had the same effect irrespective of food concentration, and that a combined model could be used to predict GPT from knowledge of food concentration and temperature (Eq. 8). The model does not require inclusion of any allometric terms, since we found non significant effects of body size on GPT (Fig. 5, Table 1). Thus, we recommend the use of Eq. (8) when no direct measurements of GPT are available. This relationship is based on laboratory experimentation which might not reflect actual field conditions, especially food type, and does not take into account the potential negative effect of large, non-ingestible diatom chains on ingestion rates (Acuña et al. 1999). Although further field validation of this model is clearly required, we have established here the basis for the measurement and prediction of gut throughput dynamics in appendicularians.

Acknowledgements. We wish to thank A. Bochdansky and D Deibel for making an early version of their manuscript available to us and for their lively discussion and criticism. Special thanks are due to $M$. Kiefer for his help during the early experimental phase of this work, and $10 \mathrm{M}$. Kieier and D. Fernández for comments on an early version of this paper. J. M. López helped in elucidating the structure of the digestive system of Oikopleura dioica. Discussions with R. Anadón and I. Huskin, and comments by 4 anonymous referees were particularly helpful. This is a contribution of the University of Oviedo to EU projects OMEX II - Phase II (MAS3-CT97-0076) to R. Anadón and EURAPP (MAS3-CT98-0161) to J.L.A.

\section{LITERATURE CITED}

Acuña JL, Anadón R (1992) Appendicularian assemblages in a shelf area and their relationship with temperature. J Plankton Res 14(9):1233-1250 
Acuña JL, Deibel D, Sooley S (1994). A simple device to transfer large and delicate planktonic organisms. Limnol Oceanogr 39(8):2001-2003

Acuña JL, Deibel D, Bochdansky AB, Hatfield E (1999). In situ ingestion rates of appendicularian tunicates in the Northeast Water Polynya (NE Greenland). Mar Ecol Prog Ser $186: 149-160$

Acuña JL, Kiefer $M$ (in press) Functional response of the appendicularian Oikopleura dioica. Limnol Oceanogr

Alldredge AL (1981) The impact of appendicularian grazing on natural food concentrations in situ. Limnol Oceanogr $26(2): 247-257$

Arashkevich YG (1977) Duration of food digestion in marine copepods. Pol Arch Hydrobiol 24 (Suppl):431-438

Arashkevich YG, Tseytlin VB (1978) Dependence of the ration on food concentration in phytophagous copepods and their feeding mechanism. Oceanology 18:347-351

Baars MA, Oosterhuis SS (1985) Zooplankton grazing in natural water with high concentration of ${ }^{14} \mathrm{C}$ bicarbonate: variable live controls and gut passage time. Hydrobiol Bull 19(1):71-80

Bochdansky AB, Deibel D (1999) Functional feeding response and behavioral ecology of Oikopleura vanhoeffeni (Appendicularia, Tunicata) J Exp Mar Biol Ecol 233:181-211

Bochdansky AB, Deibel D, Hatfield EA (1998) Chlorophyll a conversion and gut passage time for the pelagic tunicate Oikopleura vanhoeffeni (Appendicularia). J Plankton Res 20(11):2179-2197

Caparroy P, Carlotti F (1996) A model for Acartia tonsa: effect of turbulence and consequences for the related physiological processes. J Plankton Res 18(11):2139-2177

Dagg MJ, Walser WE (1987) Ingestion, gut passage, and egestion by the copepod Neocalanus plumchrus in the laboratory and in the subarctic Pacific Ocean. Limnol Oceanogr 32(1):178-188

Dam HG, Peterson WT (1988) The effect of temperature on the gut clearance rate constant of planktonic copepods. J Exp Mar Biol Ecol 123:1-14

Fenaux R (1989) Les mecanismes de l'alimentation chez les appendiculaires. Océanis 15(1):31-37

Fenaux R, Gorsky G (1985) Nouvelle technique d'elevage des appendiculaires. Rapp Comm Int Mer Médit 29(9): $291-292$

Gauld DT (1957) A peritrophic membrane in calanoid copepods. Nature 179:325-326

Gorsky G, Palazzoli 1, Fenaux R (1987) Influence of tem-

Editorial responsibility: Otto Kinne (Editor),

Oldendorf/Luhe, Germany perature changes on the oxygen uptake and ammonia and phosphate excretion, in relation to body size and weight, in Oikopleura dioica (Appendicularia). Mar Biol 94:191-201

Head EJH (1986) Estimation of Arctic copepod grazing rates in vivo and comparison with in-vitro methods. Mar Biol 92: $371-379$

Huitema BE (1980) The analysis of covariance and alternatives. Wiley-Interscience, New York

Landry MR, Lorenzen CJ, Peterson WK (1994) Mesozooplankton grazing in the Southern California Bight. II. Grazing impact and particulate flux. Mar Ecol Prog Ser 115:73-85

Mackas D, Bohrer R (1976) Fluorescence analysis of zooplankton gut contents and investigation of diel feeding patterns. J Exp Mar Biol Ecol 25:77-85

Madin LP, Cetta CM (1984) The use of gut fluorescence to estimate grazing by oceanic salps. J Plankton Res 6(3): $475-492$

Morales CE, Bautista B, Harris RP (1990) Estimates of ingestion in copepod assemblages: gut fluorescence in relation to body size. In: Barnes M, Gibson RN (eds) Trophic relationships in the marine environment. Proc 24th Europ Mar Biol Symp, Aberdeen University Press, p 565-577

Penry DL, Jumars PA (1987) Modeling animal guts as chemical reactors. Am Nat 129(1):69-96

Perissinotto R, Pakhomov EA (1996) Gut evacuation rates and pigment destruction in the Antarctic krill Euphasia superba. Mar Biol 125:47-54

Peters RH (1983) The ecological implications of body size. In: Beck E, Birks MJB, Connor EF (eds) (Cambridge Studies in ecology, Vol 2. Cambridge University Press, Cambridge

Peterson W, Painting S, Barlow R (1990) Feeding rates of Calanoides carinatus: a comparison of five methods including evacuation of the gut fluorescence method. Mar Ecol Prog Ser 63:85-92

Quade D (1967) Rank analysis of covariance. J Am Stat Assoc 62:1187-1200

Ricker WE (1984) Computation and uses of central trend lines. Can J Zool 62:1897-1905

Strathmann RR (1967) Estimating the organic carbon content of phytoplankton from cell volume or plasma volume. Limnol Oceanogr 12:411-418

Willows RI (1992) Optimal digestive investment: a model for filter feeders experiencing variable diets. Limnol Oceanogr 37(4):829-847

Submitted: January 15, 1999; Accepted: June 16, 1999 Proofs received from author(s): December 23, 1999 